\title{
Short communication Controversies in breast cancer: the mammalian target of rapamycin as a target for breast cancer therapy
}

\author{
Serena Di Cosimo
}

Breast Cancer Centre, Vall d-Hebron University Hospital, Passeig Vall d-Hebron 119-129, 08305 Barcelona, Spain

Corresponding author: Serena Di Cosimo, sdicosimo@hotmail.com

Published: 18 December 2009

This article is online at

http://breast-cancer-research.com/supplements/11/S3/S25

\section{Introduction}

The phosphatidylinositol 3-kinase (PI3K)/Akt/mammalian target of rapamycin (mTOR) pathway is a critical signaling pathway downstream of the growth factor receptor tyrosine kinases that regulates cell growth and survival. mTOR functions depend on the associated protein RAPTOR in the mTORC1 complex or RICTOR in the mTORC2 complex. Of the two complexes, mTORC1 has been the most extensively investigated as an anti-tumor target, because of its modulation by rapamycin and rapamycin analogs, such as everolimus and temsirolimus.

Specifically, mTORC1 serves as a molecular sensor to control protein synthesis via regulation of two separate downstream targets: the $40 S$ ribosomal protein S6 kinase $(p 70 S 6 K)$ and the eukaryotic initiation factor 4E binding protein-1 [1]. Upon activation, p70S6K directly phosphorylates the $40 S$ ribosomal protein S6, which correlates with enhanced translation of transcripts with a 5 '-terminal oligopyrimidine tract, such as elongation factor-1 alpha and ribosomal proteins [2]. mTOR phosphorylation of the translational repressor $4 \mathrm{E}$ binding protein-1 results in its dissociation from eukaryotic initiation factor $4 \mathrm{E}$, and in the initiation of cap-dependent translation of transcripts with long, highly structured $5^{\prime}$-untranslated regions, such as cyclin $D_{1}$ and cmyc [3]. By regulating the components of the protein synthetic machinery and cap-dependent translation, both of the mTOR-dependent phosphorylation events therefore lead to translation of proteins required for cell cycle transit.

\section{Mammalian target of rapamycin and breast cancer}

The PI3K/Akt/mTOR pathway can be activated in breast cancer by a number of mechanisms. These mechanisms include amplification of the PI3K p110 alpha catalytic subunit or Akt; mutation of the PI3K p85 beta regulatory subunit or
Breast Cancer Research 2009, 11(Suppl 3):S25

(doi:10.1186/bcr2444)

(c) 2009 BioMed Central Ltd

p70S6K; loss of the phosphatase and tensin homologue deleted on chromosome 10 (PTEN), which inhibits PI3Kdependent activation of Akt and terminates PI3K-mediated signalling; and, more often, sustained activation or overexpression of cell surface growth factor receptors, such as those of the human epidermal growth factor receptor family - HER1 or EGFR and HER2 or insulin-like growth factor-1 receptor (IGF1R) (reviewed in [4]).

The presence of compensatory pathways is not surprising in such a complicated network. One negative feedback loop involves $\mathrm{p} 70 \mathrm{~S} 6 \mathrm{~K}$, insulin-like growth factor receptor substrate 1 and IGF1R proteins. Insulin-like growth factor receptor substrate 1 is a docking protein for the IGF1R and serves to activate the regulatory subunit of PI3K. Phosphorylation of p70S6K by activated mTOR leads to the degradation of insulin-like growth factor receptor substrate 1, and consequently to suppression of PI3K/Akt signaling. The inhibition of p70S6K through mTOR-targeted agents therefore interrupts this negative feedback, resulting in sustained activation of the IGF1R signaling [5]. Taking advantage from this compensatory pathway, combined therapies designed to block both mTOR and IGF1R pathways or to block both mTOR and $\mathrm{PI} 3 \mathrm{~K} / \mathrm{Akt}$ pathways may provide a synergistic effect [6]. Other studies have demonstrated that inhibition of mTORC1 leads to mitogen-activated protein kinase pathway activation through a PI3K-dependent feedback loop [7], and that prolonged exposure to rapamycin may lead to Akt inhibition through depletion of mTORC2 (mTOR-rictor complex), which normally sits upstream and activates Akt [8].

\section{Mammalian target of rapamycin blockade}

Consistent with its critical role in cell growth and survival, the high rate of dysregulation in breast cancer [9] and in the onset of resistance to conventional anti-cancer strategies including anti-HER2 and endocrine therapy [4] - PI3K/Akt/

IGF1R = insulin-like growth factor-1 receptor; $m T O R=$ mammalian target of rapamycin; $p 70 S 6 \mathrm{~K}=40 \mathrm{~S}$ ribosomal protein $\mathrm{S} 6$ kinase; PI3K $=$ phosphatidylinositol 3-kinase. 
mTOR cascade has soon become an attractive target for drug development. Clinical trials are currently underway with PI3K, Akt and mTOR inhibitors. NVP-BEZ235 belongs to the class of imidazoquinolines, and potently and reversibly inhibits the PI3K catalytic activity by competing at its ATPbinding site. Other agents include the dual PI3K/mTOR inhibitor XL765, and the pure PI3K inhibitor XL147.

\section{Clinical studies}

Initial data from phase I studies suggest that these compounds are safe and that PI3K signaling inhibition is achievable (reviewed in [10]). The mTOR inhibitors temsirolimus and everolimus are further ahead in development either as a single agent or in combination with endocrine therapy. In the case of everolimus, a series of well-conducted pharmacokinetic/pharmacodynamic studies that analyzed mTOR inhibition in tumors as well as in surrogate tissues [11] led to the identification of a dose of $10 \mathrm{mg} /$ day as optimal for further clinical development [12,13]. In a subsequent phase II, double-blind, randomized study of everolimus in combination with letrozole versus placebo and letrozole in the neoadjuvant setting [13], the combination arm proved to be superior over letrozole and placebo with a higher significant response rate (68\% vs. 59\%) [13]. This study incorporated carefully conducted prestudy and onstudy tumor biopsies and pharmacodynamic studies, and demonstrated a near doubling of the cell cycle response rate by decreases in $\mathrm{Ki} 67$ in the everolimus group - the patients with a PI3K mutation being those with the greatest benefit. This is potentially important, since a Ki67 drop in the neoadjuvant setting has been recently demonstrated to correlate with long-term outcome.

The initial phase II study of letrozole alone or in combination with the other mTOR inhibitor temsirolimus in metastatic breast cancer patients showed benefit from the combination in terms of median progression-free survival (13.2 months vs. 11.6 months) [14]. The successive large, phase III, randomized trial of letrozole alone or in combination with temsirolimus in 992 postmenopausal women was terminated early, however, after an interim analysis demonstrated a lack of benefit from the combination [15]. This negative result is not ascribed to the lack of efficacy of this combinatorial strategy, but rather to the suboptimal inhibition of the mTOR pathway with the dose of temsirolimus implemented (30\% of patients discontinued treatment), to the study population (phase II was realized in heavily pretreated and endocrine-resistant patients, whereas phase III was up front), and to the unselected patient population, with the inability to identify patients in whom the tumors exhibit dependence on $\mathrm{PI} 3 \mathrm{~K} / \mathrm{mTOR}$ activation.

In addition, as mentioned above, evidence exists that feedback mechanisms may overcome the effects of mTOR inhibition. An understanding of compensatory pathways is therefore also critical to optimize targeted therapies. Based on preclinical models, dual inhibition of both IGF1R - with either monoclonal antibodies or tyrosine kinase inhibitors and mTOR results in a superior antiproliferative effect over each single strategy [5], and this combination is now being explored in phase I/II trials in patients with breast cancer.

Another area of intensive research is the combination of inhibitors of HER2 and mTOR inhibition. The rationale for this combination is the interaction of HER2 and the PI3K pathway, which ultimately funnels into the mTOR pathway. Based on promising early results, one phase II study is examining the safety, tolerability and response rate of the combination of trastuzumab and everolimus in patients with HER2-overexpressing breast cancer with trastuzumabrefractory metastatic disease [16].

\section{Conclusions}

The PI3K/Akt/mTOR pathway plays a critical role in breast cancer development and resistance to traditional anti-tumor strategy including endocrine-targeted and HER2-targeted agents. Treatment with mTOR inhibitors has modest activity in breast cancer patients with a response rate of $10 \%$. A combined approach has produced promising results in pretreated postmenopausal breast cancer patients and in patients harboring $\mathrm{PI} 3 \mathrm{~K}$ mutations treated with endocrine therapy. Studies are ongoing to assess the efficacy of mTOR inhibitors with chemotherapy and HER2-targeted agents to overcome resistance to the anti-Her2 monoclonal antibody trastuzumab. Consistent with a mechanistic-based approach, studies are currently ongoing with mTOR and IGF1R inhibitors and with mTOR and PI3K inhibitors to prevent the negative feedback loops secondary to mTOR inhibition.

\section{Competing interests}

The author declares that they have no competing interests.

\section{Acknowledgement}

This article has been published as part of Breast Cancer Research Volume 11 Suppl 3 2009: Controversies in Breast Cancer 2009. The full contents of the supplement are available online at http://breastcancer-research.com/supplements/11/S3.

\section{References}

1. Schmelzle T, Hall M: TOR, a central controller of cell growth. Cell 2000, 103:253-262.

2. Jefferies H, Fumagalli S, Dennis PB: Rapamycin suppresses $5^{\prime}$ TOP mRNA translation through inhibition of p70s6k. EMBO J 1997, 16:3693-3704.

3. Gingras A, Gygi S, Raught B: Regulation of 4E-BP1 phosphorylation: a novel two-step mechanism. Genes Dev 1999, 13: $1422-1437$.

4. Vivanco, I, Sawyers C: The phosphatidylinositol 3-kinase AKT pathway in human cancer. Nat Rev Cancer 2002, 2:489-501.

5. Di Cosimo S, Seoane J, Guzman M: Combination of the mammalian target of rapamycin (mTOR) inhibitor everolimus (E) with the insulin like growth factor-1-receptor (IGF-1-R) inhibitor NVP-AEW-541: a mechanistic based anti-tumor strategy. J Clin Oncol 2005, 23(Suppl 16):3112.

6. Di Cosimo S, Scaltriti M, Val D: The PI3-K/AKT/mTOR pathway as a target for breast cancer therapy. J Clin Oncol 2007, 25 (Suppl 18):3511.

7. Carracedo A, Ma L, Teruya-Feldstein J: Inhibition of mTORC1 leads to MAPK pathway activation through a PI3K-dependent feedback loop in human cancer. $J$ Clin Invest 2008, 118:3065-3074. 
8. Sarbassov DD, Ali SM, Sengupta S: Prolonged rapamycin treatment inhibits mTORC2 assembly and Akt/PKB. Mol Cell 2006, 22:159-168.

9. Stemke-Hale K, Gonzalez-Angulo AM, Lluch A: An integrative genomic and proteomic analysis of PIK3CA, PTEN, and AKT mutations in breast cancer. Cancer Res 2008, 68:6084-6091.

10. Markman B, Atzori F, Pérez-García J: Status of PI3K inhibition and biomarker development in cancer therapeutics. Ann Oncol 2009, in press (epub ahead of print).

11. Tabernero J, Rojo F, Calvo E: Dose- and schedule-dependent inhibition of the mammalian target of rapamycin pathway with everolimus: a phase I tumor pharmacodynamic study in patients with advanced solid tumors. J Clin Oncol 2008, 26: 1603-1610.

12. Ellard SL, Clemons M, Gelmon KA: Randomized phase II study comparing two schedules of everolimus in patients with recurrent/metastatic breast cancer: NCIC Clinical Trials Group IND.163. J Clin Oncol 2009, 27:4536-4549.

13. Baselga J, Semiglazov V, van Dam P: Phase II randomized study of neoadjuvant everolimus plus letrozole compared with placebo plus letrozole in patients with estrogen receptor-positive breast cancer. J Clin Oncol 2009, 27:2630-2637.

14. Baselga J, Fumoleau P, Gil M: Phase II, 3 arm study of CCl-779 in combination with letrozole in postmenopausal women with locally advanced or metastatic breast cancer. Preliminary results. J Clin Oncol 2004, 22(Suppl 14):544.

15. Chow LWS, Sun Y, Jassem J: Phase 3 study of temsirolimus with letrozole or letrozole alone in postmenopausal women with locally advanced or metastatic breast cancer. Breast Cancer Res Treat 2006, 100(Suppl 1):6091.

16. André $F$, Campone M, Hurvitz SA: Multicenter phase I clinical trial of daily and weekly RAD001 in combination with weekly paclitaxel and trastuzumab in patients with HER2-overexpressing metastatic breast cancer with prior resistance to trastuzumab. J Clin Oncol 2008, 26(Suppl 20):1003. 